\title{
Finite Sample Properties of Parameterized Expectations Algorithm Solutions; Is the Length So Determinant?
}

\author{
A. Jesús Sánchez-Fuentes \\ Complutense Institute of International Studies (ICEI-UCM) \& GEN-UVigo (Spain) \\ Received 22 December 2020 | Accepted 10 February 2022 | Published 25 February 2022
}

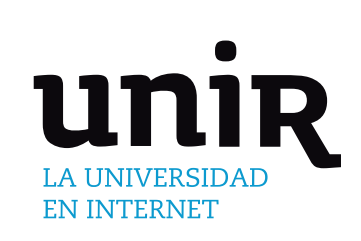

ABSTRACT

The solution of the Parameterized Expectations Algorithm (PEA) is well defined based on asymptotic properties. In practice, it depends on the specific replication of the exogenous shock(s) used for the resolution process. Typically, this problem is reduced when a sufficiently long replication is considered. In this paper, we suggest an alternative approach which consists of using several, shorter replications. A centrality measure (the median) is used then to discriminate among the different solutions using two different criteria, which differ in the information used. On the one hand, the distance to the vector composed by median values of PEA coefficients is minimized. On the other hand, distances to the median impulse response is minimized. Finally, we explore the impact of considering alternative approaches in an empirical illustration.

\section{KEYWORDS}

\author{
Nonlinear Models, \\ Numerical Solution \\ Methods, Optimal \\ Growth, Parameterized \\ Expectations Algorithm. \\ DOI: $10.9781 /$ ijimai.2022.02.007
}

\section{INTRODUCTION}

$\mathrm{T}^{\mathrm{H}, \mathrm{s}}$ HE Parameterized Expectations Algorithm (PEA) is a widely applied method for solving nonlinear stochastic dynamic models with rational expectations (see [1]-[8]) The PEA scheme involves approximating the conditional expectation functions in the Euler equations with certain parametric functions, and the use of a numerical optimization method to estimate parameter values.

A common problem with the PEA is that the solutions are obtained from a specific replication of exogenous random process(es). Due to the asymptotic properties of the algorithm and increasing computational costs, authors typically have considered only one replication of the exogenous shocks but with a sufficiently long simulation length. However, as [9] pointed out: "... it may be necessary to use an extremely long simulation in order to obtain the same fitted coefficients of the approximating function across replications of the simulation”. By contrast, [10] claim that only quantitative differences are observed (qualitative properties remain). In any event, they all agree that basing one's conclusions on a "non-unique" solution may cause results to be less robust than otherwise.

Contrary to [9] proposal which involves a parallel implementation of the PEA algorithm that enables a sufficiently long replication, we adopt a different approach consisting of sampling a sufficient number of shorter replications, in the framework of a Montecarlo experiment. A centrality measure (the median) is used to discriminate among the different solutions. The criteria differ in the information used. On the one hand, the distance to the vector composed by median values of PEA coefficients is minimized. On the other hand, distances to the median impulse response is minimized.

\footnotetext{
* Corresponding author.

E-mail address: ajsanchezfuentes@ucm.es
}

We consider two models to frame the discussion: the simple neoclassical growth model, and the Cooley and Hansen (1989) model, that adds to the previous model a non-convexity, indivisible labour and introduces money via a cash-in-advance constraint in consumption.

Our main conclusion is that the criterion choosing the replication closest to the median impulse-response function appears to be the most suitable criterion for several reasons: (i) there is no bias in using shorter simulations which allows one to face much lower computational costs, (ii) it shows how different solutions behave when a transitory/permanent shock is applied (qualitative robustness is indirectly checked), and (iii) it provides a band of confidence around the final choice (the level of uncertainty is measured). Additionally, summary statistics may be obtained from the distribution of estimated coefficients.

\section{THe PEA}

Consider an economy characterized by a vector of $\mathbf{n}$ endogenous variables, $\mathbf{z}_{\mathbf{t}}$, and by a vector of $\mathbf{s}$ exogenously given shocks, $\mathbf{u}_{\mathbf{t}}$. Let the process $\left\{\mathbf{z}_{\mathbf{t}}, \mathbf{u}_{\mathrm{t}}\right\}$ be represented by a system

$$
g\left(E_{t}\left[\phi\left(z_{t+1}, z_{t}\right)\right], z_{t}, z_{t-1}, u_{t}\right)=0 \text {, for all } t
$$

where $g: \mathbb{R}^{m} x \mathbb{R}^{n} x \mathbb{R}^{n} x \mathbb{R}^{s} \rightarrow \mathbb{R}^{q}$ and $\phi: \mathbb{R}^{2 n} \rightarrow \mathbb{R}^{m}$; the vector $\mathbf{z}_{\mathbf{t}}$ includes all endogenous and exogenous variables that are inside the expectation, and $\mathbf{u}_{\mathbf{t}}$ follows a first-order Markov process. It is assumed that $\mathbf{z}_{\mathbf{t}}$ is uniquely determined by (1) if the rest of arguments are given. The functions $\mathbf{g}(\cdot)$ and $\phi(\cdot)$ are known functions once the structural parameters of the economy are fixed. Alternatively, let the solution be expressed as a law of motion $\mathbf{h}$ such that the vector $\mathbf{z}_{\mathbf{t}}$ generated by $z_{t}=h\left(z_{t-1}, u_{t}\right)$ fulfills (1), given that all past information relevant to forecast $\phi\left(z_{t+1}, z_{t}\right)$ can be summarized in a finite-dimension function of $\left\{\mathbf{z}_{\mathbf{t}-1}, \mathbf{u}_{\mathbf{t}}\right\}$.

Obtaining a solution to (1) using PEA consists of finding a

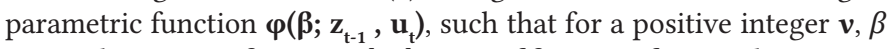
$\in \mathbb{D}^{v}$, where $\mathbb{D}^{v} \subset\left\{\beta \in \mathbb{R}^{\infty}: i\right.$-th element of $\beta$ is zero if $\left.i>v\right\}$, the process 
$\left\{\mathbf{z}_{\mathbf{t}}(\boldsymbol{\beta})\right\}$ satisfies for all $t$ the set of equations

$$
g\left(E_{t}\left[\psi\left(\beta ; z_{t-1}, u_{t}\right)\right], z_{t}(\beta), z_{t-1}(\beta), u_{t}\right)=0
$$

and the order of $\boldsymbol{v}$ is such that when solving $G(\beta)=\underset{\beta \in \mathbb{D}^{v}}{\arg \min }$ $E_{t}\left[\phi\left(z_{t+1}(\beta), z_{t}(\beta)\right)-\psi\left(\beta ; z_{t-1}(\beta), u_{t}\right)\right]^{2}$, then $\beta=G(\beta)$. This problem is solved by use of the following Gauss-Newton updating rule: $\beta^{i}=\beta^{i-1}+\lambda G\left(\beta^{i-1}\right)$ at each iteration $i$. Given these conditions, the stochastic process $\left\{\mathbf{z}_{\mathrm{t}}(\boldsymbol{\beta})\right\}$ is the PEA approximated solution. Under certain regularity conditions over the functions defining the equilibrium in (1), the function $\mathbf{g}(\cdot)$ is invertible in its second argument, and equation (2) can be written as (see [3])

$$
z_{t}(\beta)=h_{\beta}\left(z_{t-1}(\beta), u_{t}\right)
$$

for stationary and ergodic processes. [11] shows that under those regularity conditions, fulfilled by standard business cycle models, it is always possible to find an approximated function $\mathbf{h}_{\beta}(\cdot)$ arbitrarily close to the true law of motion of the system $\mathbf{h}(\cdot)$. Under the true law of motion $h\left(z_{t-1}, u_{t}\right)$, the true process $\left\{\mathbf{z}_{t}, \mathbf{u}_{t}\right\}_{-\infty}^{+\infty}$ verifying (1) is stationary. For the approximation to be acceptable, given initial conditions $\left\{\mathbf{z}_{0}, \mathbf{u}_{0}\right\}$ and an initial vector $\boldsymbol{\beta}$, the resulting process $\left\{\mathbf{z}_{\boldsymbol{t}}(\boldsymbol{\beta})\right\}_{t=1}^{T}$ verifying (2) has to be stationary.

The PEA as presented in [2] can be written as follows:

- Step 1. Fix initial conditions $\mathbf{u}_{\mathbf{0}}$ and $\mathbf{z}_{0}$; draw and fix a random series $\left\{\mathbf{u}_{\boldsymbol{t}}\right\}_{t=1}^{T}$ from a given definition. Replace the conditional expectation in (1) with a function $\varphi\left(\boldsymbol{\beta} ; \mathbf{z}_{\mathrm{t}-1}, \mathbf{u}_{\mathrm{t}}\right)$ and compute $\mathbf{z}_{t}(\boldsymbol{\beta})$ from (3).

- Step 2. For a given $\beta \in \mathbb{D}^{v}$ recursively calculate $\left\{\mathbf{z}_{t}(\boldsymbol{\beta})\right\}_{t=1}^{T}$ according to $z_{t}(\beta)=h_{\beta}\left(z_{t-1}(\beta), u_{t}\right)$, if $\underline{z} \leq z_{t}(\beta) \leq \bar{z}$

- Step 3. Find a $\mathbf{G}(\boldsymbol{\beta})$ that satisfies $G(\beta)=\underset{\beta \in \mathbb{D}^{\nu}}{\arg \min }$ $E_{t}\left[\phi\left(z_{t+1}(\beta), z_{t}(\beta)\right)-\psi\left(\beta ; z_{t-1}(\beta), u_{t}\right)\right]^{2}$. In order to perform this step, one can run a nonlinear least squares regression with the sample $\left\{z_{t}(\beta), u_{t}\right\}$, taking $\phi\left(z_{t+1}(\beta), z_{t}(\beta)\right)$ as a dependent variable, $\varphi(\cdot)$ as an explanatory function, and $\xi$ as a parameter vector to be estimated.

- Step 4. Compute the vector $\boldsymbol{\beta}^{\mathbf{i}+\mathbf{1}}$ for the next iteration, $\beta^{i+1}=\beta^{i}+$ $\lambda G\left(\beta^{i}\right), \lambda \in(0,1)$

Iterate on Steps $2-4$ until $\left\|\beta^{i+1}-\beta^{i}\right\|$ is below a certain tolerance value, for all $t$.

\section{The Models}

For simplicity, and without loss of generality, we have selected two quite standard models to address the discussion: the one-sector stochastic growth model (SN henceforth) and the Cooley and Hansen model ( $\mathrm{CH}$ henceforth) presented in [12]. Consider firstly the SN model,

$$
\max _{\left\{c_{t}, k_{t}\right\}_{t=0}^{\infty}} E_{0} \sum_{t=0}^{\infty} \delta^{t} \frac{c_{t}^{1-\gamma}-1}{1-\gamma}, \text { s.t. } c_{t}+k_{t}=(1-d) k_{t-1}+\theta_{t} k_{t-1}^{\alpha}
$$

where $\left.\log \theta_{t}=\rho \log \theta_{t-1}\right)+\epsilon_{t}$ with $\epsilon_{t} \sim \mathcal{N}\left(0, \sigma^{2}\right)$, the initial condition $\left(k_{-1}, \theta_{0}\right)$ is given. $\mathbf{c}_{\mathrm{t}}$ is consumption at time $t, \mathrm{k}_{\mathrm{t}-1}$ the beginning of period $t$ capital stock, $\mathbf{0}<\boldsymbol{\delta}<\mathbf{1}$ is the subjective discount factor, $\mathbf{0}<\boldsymbol{\alpha}<\mathbf{1}$ the capital share in production, $\mathbf{0}<\mathbf{d}<\mathbf{1}$ the depreciation rate, and $\mathbf{0}<\boldsymbol{\rho}<\mathbf{1}$. But for the case with logarithmic utility, $\gamma=1$, and full depreciation of capital, $\mathbf{d}=\mathbf{1}$, a closed-form solution to this model is not known. Following [4], we approximate the conditional expectation by

$$
E_{t}\left[c_{t+1}^{-\gamma}\left(1-d+\alpha \theta_{t-1} k_{t-1}^{\alpha}\right)\right] \cong \exp \left(\beta_{0}+\beta_{1} \log \theta_{t}+\beta_{2} \log k_{t-1}\right)
$$

where $\beta=\left(\beta_{0}, \beta_{1}, \beta_{2}\right)$ is a vector of coefficients to be found. To simulate the model, parameter values are fixed as:

$$
\alpha=0.33, \delta=0.95, d=0.02, \rho=0.95, \sigma=0.01, k_{-1}=k_{s S}
$$

(the subscript $s s$ refers to the steady-state values) and $\theta_{0}=1$.

The $\mathrm{CH}$ model is slightly more complex in that it includes a nonconvexity, indivisible labour. Money is introduced via a cash-inadvance constraint in consumption. The competitive equilibrium is non-Pareto-optimal and the second welfare theorem does not apply. The representative firm solves a standard profit maximization problem, while households seek to maximize their time preferences subject to their holdings of money balances and a set of standard budget constraints. There are two sources of uncertainty in this economy: an autoregressive shock to technology, $\theta_{\mathrm{t}}$, and an autoregressive logged money growth rate, $\log g_{t}$. Reference [3] preferred specification for the approximating function $\varphi(\cdot)$ to the expectation term is a third-order polynomial such that,

$$
\begin{aligned}
E_{t}\left[c _ { t + 1 } ^ { - \gamma } \left(+\alpha \theta_{t-1} k_{t-1}^{\alpha}\right.\right. & \left.\left.N_{t-1}^{1-\alpha}+1-d\right)\right] \\
& \cong \exp \left(\beta_{0}+\beta_{1} \log k_{t-1}+\beta_{2} \log \theta_{t}+\beta_{3} \log g_{t}\right. \\
& +\beta_{4}\left(\log k_{t-1}\right)^{2} \\
& +\beta_{5} \log k_{t-1} \log \theta_{t} \\
& \left.+\beta_{6}\left(\log \theta_{t}\right)^{2}+\beta_{7}\left(\log \theta_{t}\right)^{3}\right)
\end{aligned}
$$

where $\boldsymbol{\mu}_{t}$ is the Lagrange multiplier attached to the household's budget constraint, and $\mathrm{N}$ denote hours worked. Following [3], we will adopt as baseline parameterization:

$$
\begin{gathered}
\alpha=0.36, \delta=0.99, d=0.025, \rho=0.95, \rho_{g}=0.48, \\
\sigma_{\epsilon_{g}}=0.009, \sigma=0.00721, g_{s s}=1.15, A_{N}=2.86
\end{gathered}
$$

\section{Selection Criteria Between PEA Solutions}

Potential criteria should rely on the statistical properties of distributions obtained from the simulation of the models: estimated coefficients, simulated variables or impulse-response functions. Prior intuitions are next discussed. Firstly, how each solution behaves cannot be determined using the simulated series as they depend on the replication used.

Secondly, it looks reasonable, in line with studies in the field of the functional analysis facing similar problems (see [13]), to choose methods that reduce the probability of obtaining an "extreme" (less representative) solution: that with highest or lowest values of the reference distribution. Thus, commonly used concepts of centrality within a distribution may be considered. In this respect, we use the concept of median but our conclusions are robust to changes in the chosen measure. ${ }^{1}$

Thirdly, general or model-specific criteria based on economic theory can be applied as well. A chosen replication should verify some meaningful constraint(s) dictated by economic theory. Impulseresponse functions are typically used to determine solutions behaviour. As an instance, in the context of the SN model, [10] discusses the convenience of a monotonic response of consumption to a technology shock (pp. 12-13).

Fourthly, note that values coming from different replications do not represent a PEA solution. Therefore, choosing independently ideal values is not an option and a compromised choice should be done.

In the light of previous arguments, we suggest the following selection criteria:

\section{- Minimum distance to median coefficients}

First, we compute a vector composed by the median value for each coefficient $(\tilde{\beta})$. Next, we compute the distance of each vector

\footnotetext{
Results remain if distances are computed with respect to either average values or the concept of statistical depth (see [16]).
} 
of coefficients to the reference vector. Finally, the replication minimizing this distance, $\widetilde{\beta}_{\tilde{r}}$, is chosen. Analytically,

$$
r_{\tilde{\beta}}^{\min }=\min _{r=1, \ldots, R}\left\|\tilde{\beta}-\beta^{r}\right\|
$$

where $\beta^{r}$ is the coefficient vector of replication $r$.

\section{- Minima distance to median impulse response}

First, we compute the median impulse response, $\{\widetilde{I R}\}$, as a vector composed by the median value of different responses at period $h, \widetilde{r}_{h}$, $(h=1, \ldots, H)$. Next, we compute the distance of each response to the reference response and, again, we choose those minimizing it. Analytically,

$$
r_{\widetilde{I R}}^{\min }=\min _{r=1, \ldots, R}\left\|\widetilde{I R}-I R^{r}\right\|
$$

where $\widetilde{I R}=\left\{\widetilde{r}_{h}\right\}_{h=1}^{H}$ and $I R^{r}=\left\{i r_{h}\right\}_{h=1}^{H}$ is the vector composed by the response at period $h,(h=1, \ldots, H)$ of replication $r, i r_{h}^{r}$.

Within this category, we distinguish between transitory $\left(r_{\overline{I R}_{t}}^{\min }\right)$ and permanent $\left(r_{I_{R}}^{\min }\right)$.

We consider previous criteria to discriminate among solutions. Consequently, we may define $r_{\tilde{\beta}}^{\max }, r_{\overline{I R}_{t}}^{\max }$ and $r_{\overline{I R}_{p}}^{\max }$ as the replications which respectively maximize the corresponding distances. If no significant differences are observed between both choices ( $\mathrm{min}$ and max), we conclude that the criterion becomes non-informative.

These criteria are systematically checked in our Monte Carlo experiment. We consider 250 independent draws of exogenous shocks of varying size $\mathbf{T}$ for each model. We choose $\mathbf{T} \in\{1000,10000,20000$, $30000,40000,50000\}$ with the aim of checking progressively the gains obtained from increases in the simulation length. ${ }^{2}$

As a convergence criterion, we use the $\mathrm{L}^{2}$-distance between the $\boldsymbol{\beta}$-vectors obtained in two subsequent iterations be less than $10^{-5}$. With respect to the initialization of the algorithm, to the light of results shown in [14], we consider the approach suggested by [15].

\section{Results And Discussion}

Table I presents the number of the simulation (among the 250 independent draws) chosen according to each criteria. It can be observed that the different criteria rarely agree. Indeed, the only coincidence is referred to the replication maximizing $\mathrm{L}^{2}$ distance to reference values obtained in the case of $\mathrm{CH}$ model. Therefore, in order to formulate a final proposal, we are forced to discriminate in this section between the different criteria suggested before.

Table II includes summary statistics which allow us to explore, firstly, the relevance of the central issue of this paper (to determine whether there are significant differences between different PEA solutions) and, secondly, how the selection criteria exposed above perform in the cases of the $\mathrm{SN}$ and $\mathrm{CH}$ models.

The main conclusion is that distributions are much more similar for simulation lengths greater than 1,000. A decreasing trend in $\sigma_{\mathrm{K}}$ is also observed when $\mathbf{T}$ grows, in line with the asymptotic properties of this algorithm, but this parameter is mostly constant within each distribution.

Furthermore, there are significant differences regarding the cross correlation between the responses of central variables of the $\mathrm{CH}$ model. By contrast, the SN model shows no big differences among the solutions. This latter result indicates the relevance of considering selection criteria when the complexity of model raises. Additionally, selection criteria based on impulse-responses achieve reasonable (and stable) values for all the simulation lengths considered -they are in the

2 Indeed, a wider grid of lengths has been explored. For the sake of brevity, only results relative to those mentioned here are shown. range achieved for the longest distribution of simulations- whereas criterion based on median coefficients satisfy this condition only from $T=10,000$.

TABLE I. Chosen Replications. $r_{C}^{\min } / r_{C}^{\max }$ Refer to Replications Minimizing/Maximizing L L Distance to Reference Values $\left(C=\tilde{\beta}, \widetilde{R}_{t}\right.$ and $\left.\widetilde{I R_{p}}\right)$

Choices minimizing $\mathrm{L}^{2}$ distance to reference values

\begin{tabular}{|l|c|c|c|c|c|c|}
\hline \multirow{2}{*}{$\begin{array}{l}\text { Replication } \\
\text { length }\end{array}$} & \multicolumn{3}{|c|}{ SN model } & \multicolumn{3}{c|}{ CH model } \\
\cline { 2 - 7 } & $\tilde{\beta}$ & $\widetilde{I R}_{t}$ & $\widetilde{I R}_{p}$ & $\tilde{\beta}$ & $\widetilde{I R}_{t}$ & $\widetilde{I R}_{p}$ \\
\hline 1,000 & 129 & 236 & 13 & 27 & 240 & 219 \\
\hline 10,000 & 68 & 80 & 51 & 54 & 247 & 40 \\
\hline 20,000 & 221 & 226 & 161 & 92 & 111 & 95 \\
\hline 30,000 & 158 & 151 & 164 & 38 & 93 & 136 \\
\hline 40,000 & 125 & 71 & 32 & 27 & 111 & 154 \\
\hline 50,000 & 99 & 200 & 201 & 71 & 149 & 48 \\
\hline
\end{tabular}

Choices maximizing $\mathrm{L}^{2}$ distance to reference values

\begin{tabular}{|l|c|c|c|c|c|c|}
\hline \multirow{2}{*}{$\begin{array}{l}\text { Replication } \\
\text { length }\end{array}$} & \multicolumn{3}{|c|}{ SN model } & \multicolumn{3}{c|}{ CH model } \\
\cline { 2 - 7 } & $\tilde{\beta}$ & $\widetilde{I R}_{t}$ & $\widetilde{I R_{p}}$ & $\tilde{\beta}$ & $\widetilde{I R}_{t}$ & $\widetilde{I R_{p}}$ \\
\hline 1,000 & 41 & 41 & 41 & 25 & 199 & 199 \\
\hline 10,000 & 115 & 21 & 217 & 6 & 51 & 51 \\
\hline 20,000 & 195 & 41 & 184 & 154 & 98 & 98 \\
\hline 30,000 & 18 & 57 & 7 & 32 & 143 & 143 \\
\hline 40,000 & 57 & 198 & 30 & 44 & 117 & 117 \\
\hline 50,000 & 195 & 212 & 91 & 195 & 94 & 44 \\
\hline
\end{tabular}

Notes: (1) Monte Carlo experiment: (a) 250 independent replications are computed for each length and model, (b) L2 distance between subsequent vectors obtained is required to be less than $10^{-5}$, (c) Reference [15] approaches is used to initialise the algorithm. (2) Figures indicate the number of the simulation (among the 250 independent draws) chosen according to each criteria.

We further look in detail how the values of the coefficients change according to each replication length and selection criteria. Fig. 1 presents the distribution of coefficients for the SN model, and Fig. 2 those of the $\mathrm{CH}$ model. On each box, the central mark is the median, the edges of the box are the $25^{\text {th }}$ and $75^{\text {th }}$ percentiles, the whiskers extend to the most extreme data points not considered outliers, and outliers are plotted individually. Moreover, solutions minimizing and maximizing distance to reference values of different criteria selected above are remarked.

Some conclusions can be drawn: (i) we confirm again, from a different perspective, that the variance is reduced when the replication length is increased. The gain loses importance for lengths higher than 20000. (ii) Median values of coefficients are almost equal for lengths higher than $10000 .^{3}$ (iii) The criteria based on coefficients and those based on median responses are not coincident (as we commented before) in the sense that those based on median responses do not clearly discriminate between choices minimizing and maximizing distance within the distribution of the coefficients. (iv) The criterion based on median coefficients is more precise on those with a high variability. Otherwise, choices may be further away from the corresponding median value. As an instance, see coefficients $\boldsymbol{\beta}_{8}$ and $\boldsymbol{\beta}_{4}$ of the $\mathrm{CH}$ model.

Fig. 3, Fig. 4, Fig. 5 and Fig. 6 show the responses of $k$ (capital in both models) to a transitory/permanent technology shock for each simulation length and model. Additionally, replications minimizing/ maximizing $L^{2}$ distance to reference values of different criteria are remarked. ${ }^{4}$

\footnotetext{
${ }^{3}$ Tests statistics confirm this finding. The results are available upon request.

${ }^{4}$ For the sake of simplicity, criteria based on different types of shocks are not compared within the same graph.
} 
TABLE II. Summary Statistics. $r_{c}^{\min } / r_{c}^{\max }$ Refer to Replications Minimizing/Maximizing L ${ }^{2}$ Distance to Reference Values $\left(C=\widetilde{\beta}, \widetilde{I R_{t}}\right.$ and $\left.\widetilde{I R_{p}}\right)$

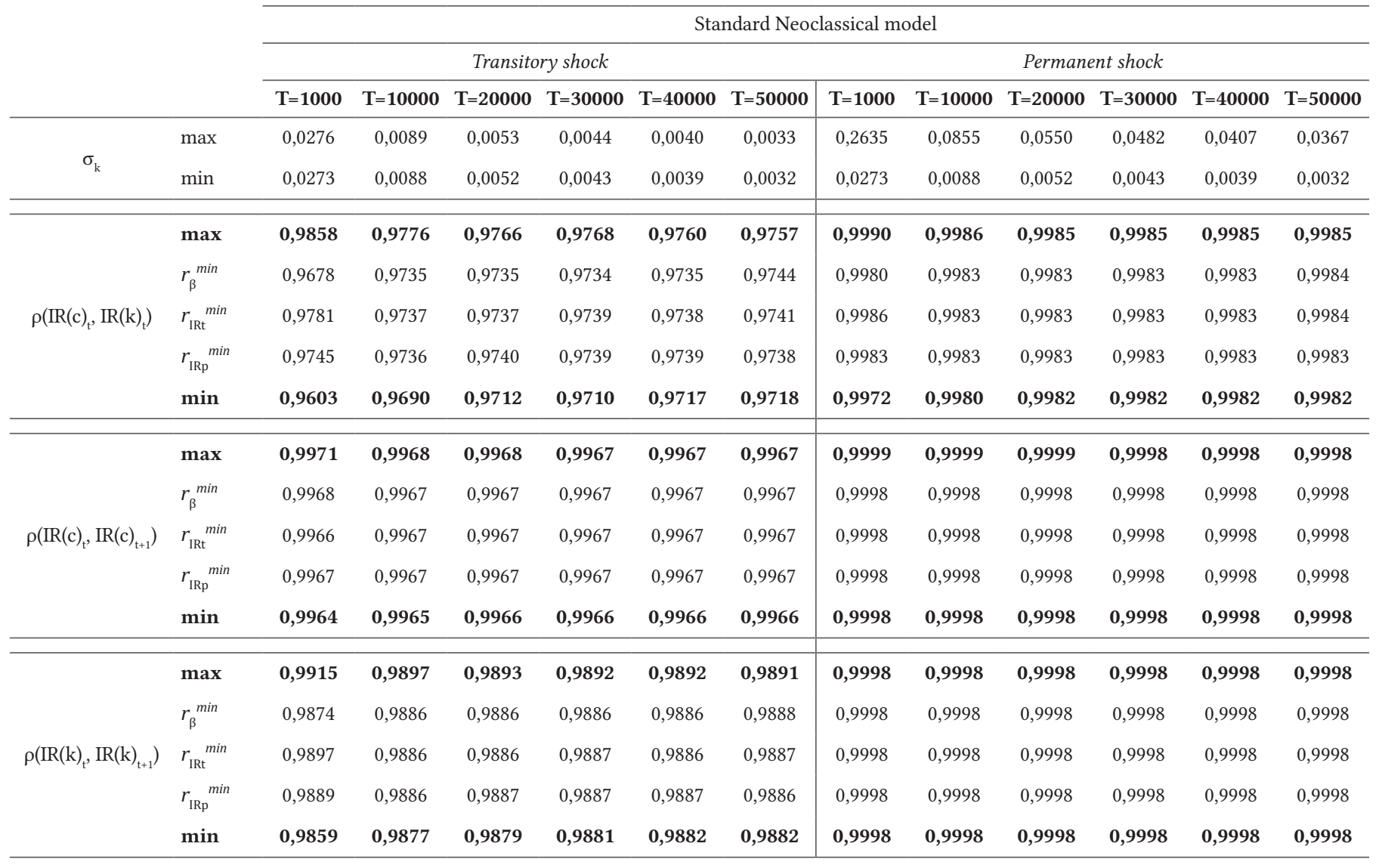

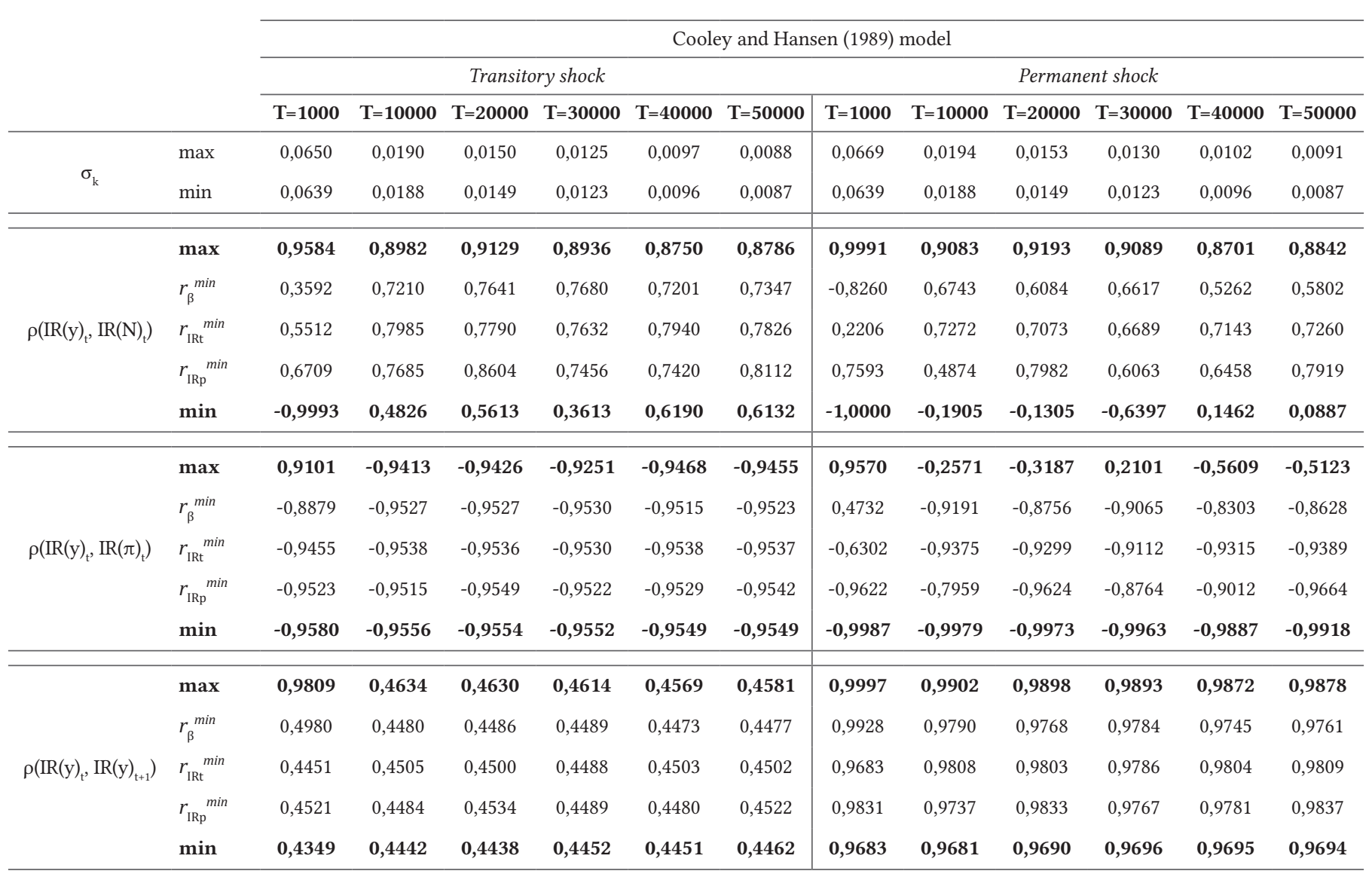



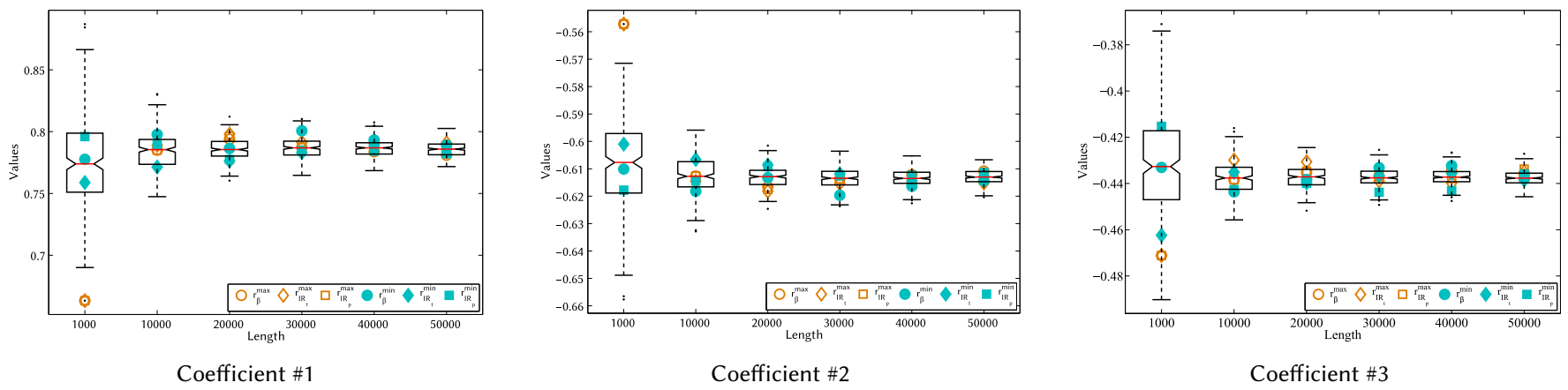

Fig. 1. Boxplots of functional form coefficients of parameterized expectations. SN model. $r_{C}^{\min } / r_{C}^{\max }$ refer to replications minimizing/maximizing $\mathrm{L}^{2}$ distance to reference values $\left(C=\tilde{\beta}, \widetilde{R}_{t}\right.$ and $\widetilde{R_{p}}$ ). (1) Monte Carlo experiment: (a) 250 independent replications are computed for each length and model, (b) L2 distance between subsequent $\beta$-vectors obtained is required to be less than 10-5, (c) Reference [15] approach is used to initialise the algorithm.

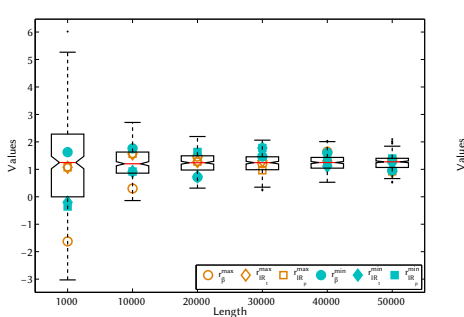

Coefficient \#1

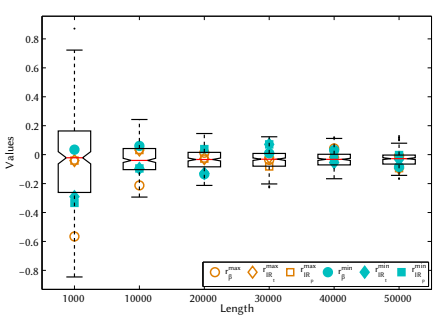

Coefficient \#5

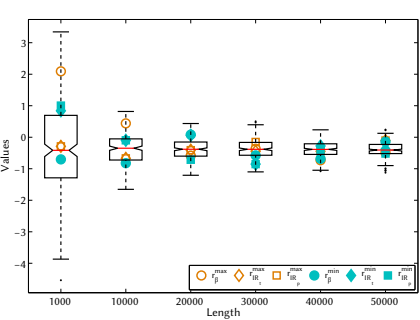

Coefficient \#2

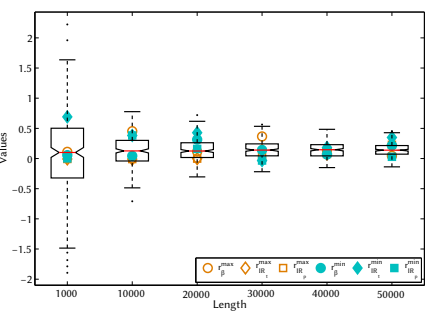

Coefficient \#6

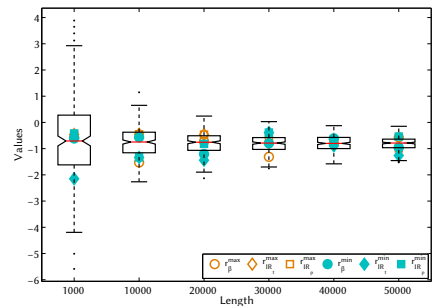

Coefficient \#3

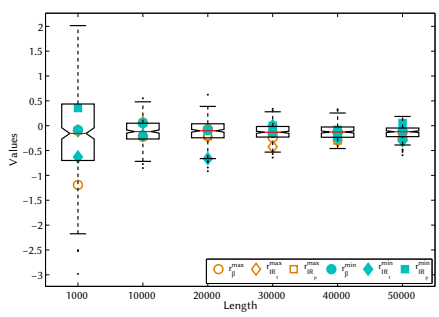

Coefficient \#7

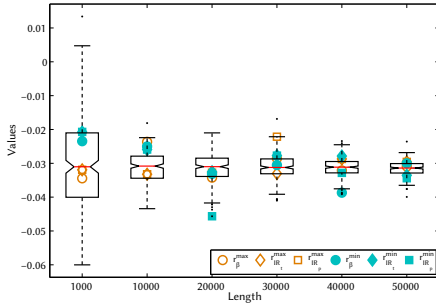

Coefficient \#4

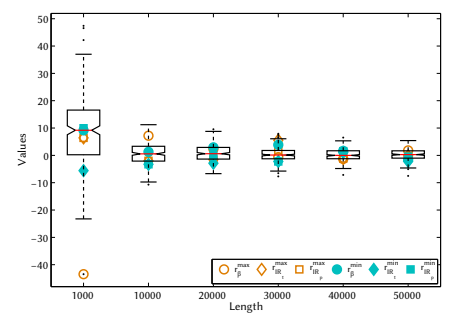

Coefficient \#8

Fig. 2. Boxplots of functional form coefficients of parameterized expectations. $\mathrm{CH}$ model. $r_{C}^{\min } / r_{C}^{\max }$ refer to replications minimizing $/$ maximizing $\mathrm{L}^{2}$ distance to reference values $\left(C=\tilde{\beta}, \widetilde{R}_{t}\right.$ and $\left.\widetilde{R}_{p}\right)$. (1) Fig. 1. notes applies here.

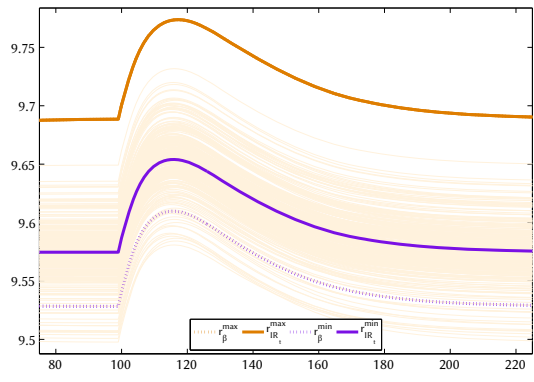

Length $=1,000$

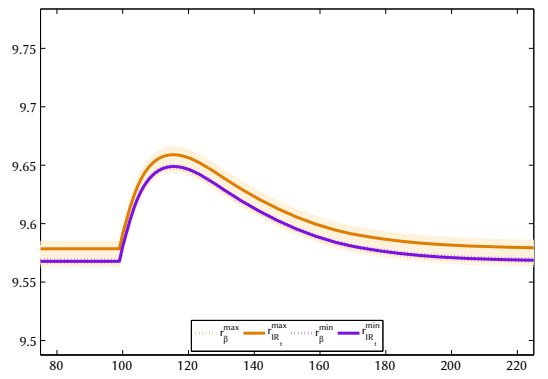

Length $=30,000$

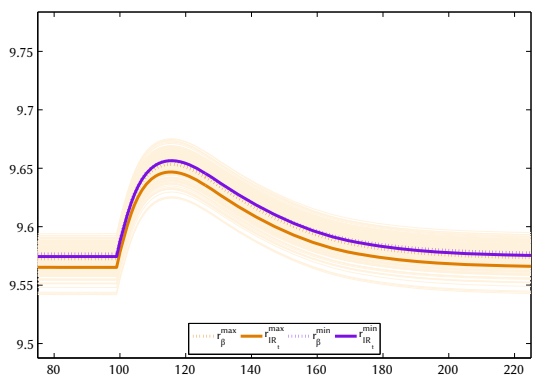

Length $=10,000$

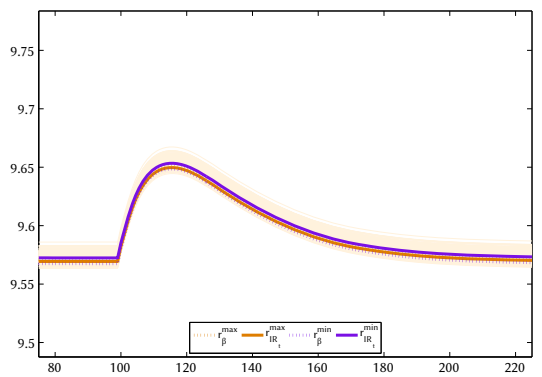

Length $=40,000$

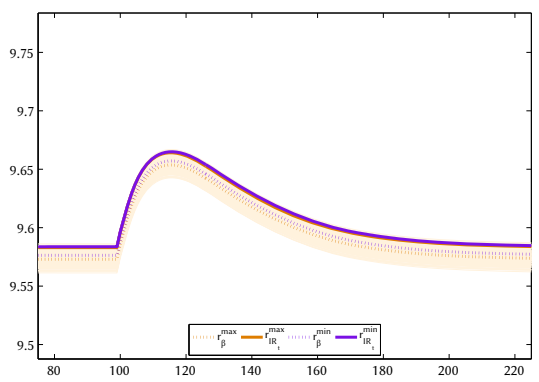

Length $=20,000$

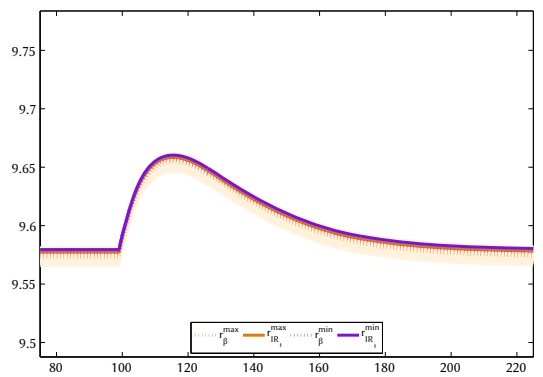

Length $=50,000$

Fig. 3. Responses of $\mathrm{k}$ to a transitory technology shock. SN model. $r_{C}^{\min } / r_{C}^{\max }$ refer to replications minimizing/maximizing $\mathrm{L}^{2}$ distance to reference values $\left(C=\tilde{\beta}, \widetilde{R}_{t}\right.$ and $\left.\widetilde{R}_{p}\right) .(1)$ Fig. 1. notes applies here. 

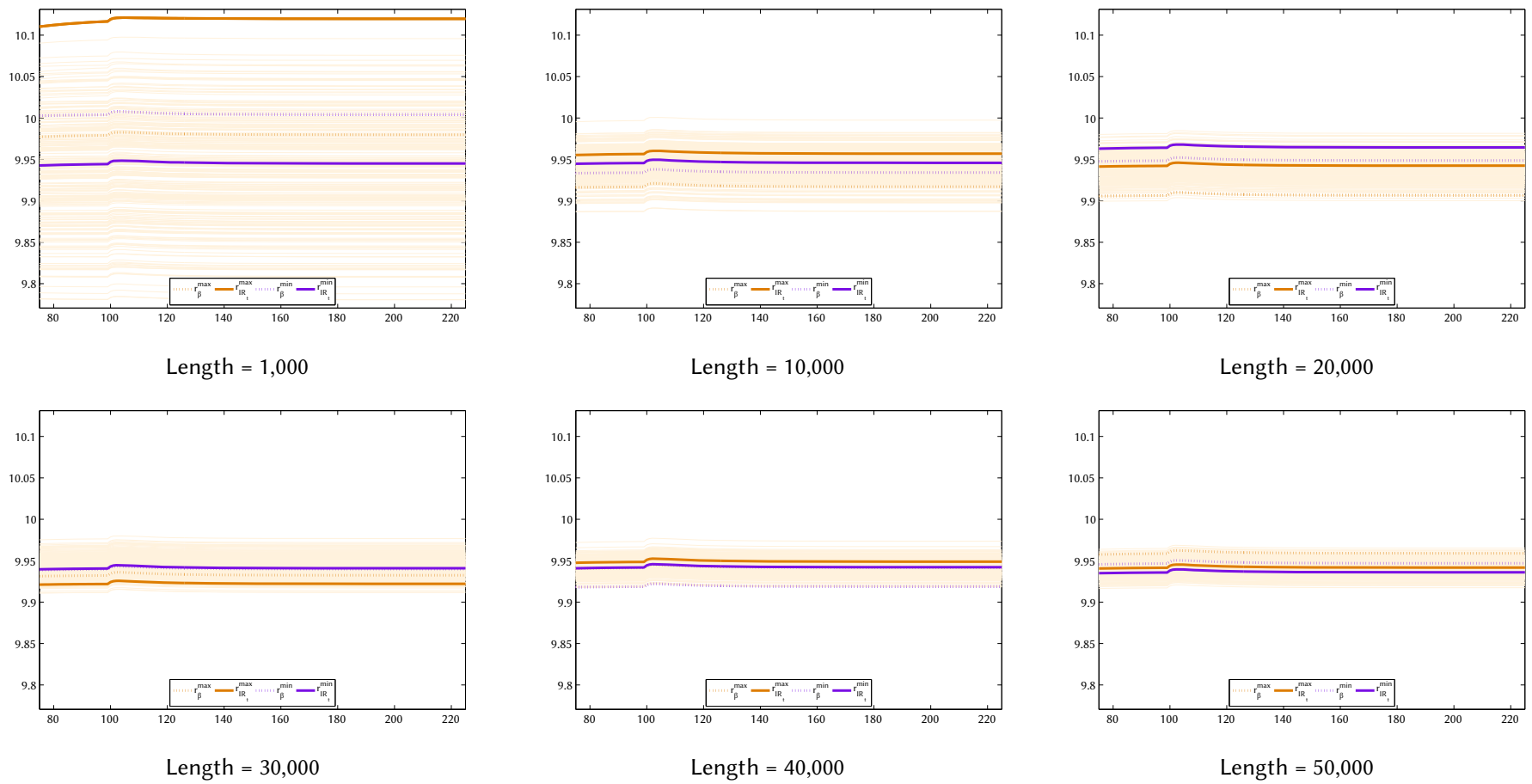

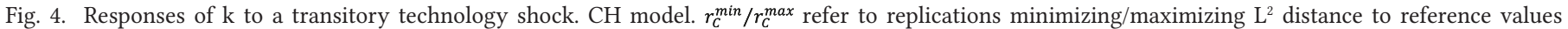
$\left(C=\tilde{\beta}, \widetilde{R}_{t}\right.$ and $\left.\widetilde{I R_{p}}\right)$. (1) Fig. 1. notes applies here.

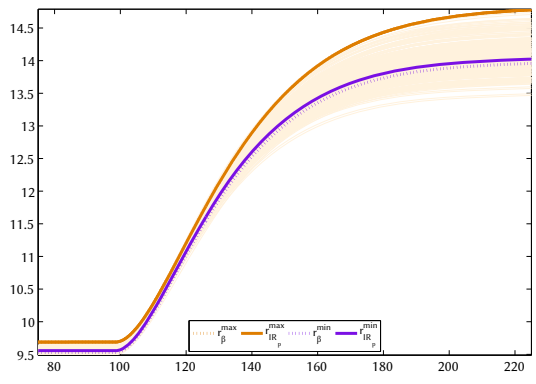

Length $=1,000$

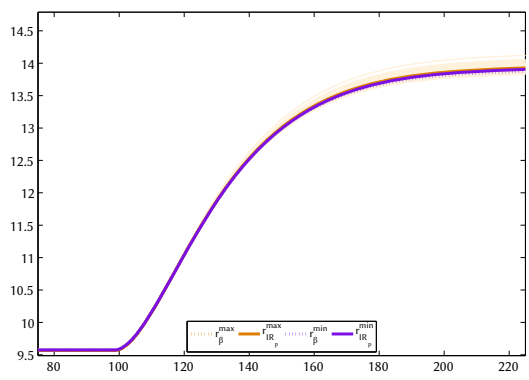

Length $=30,000$

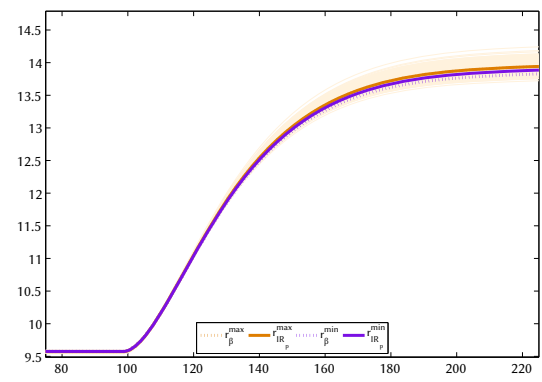

Length $=10,000$

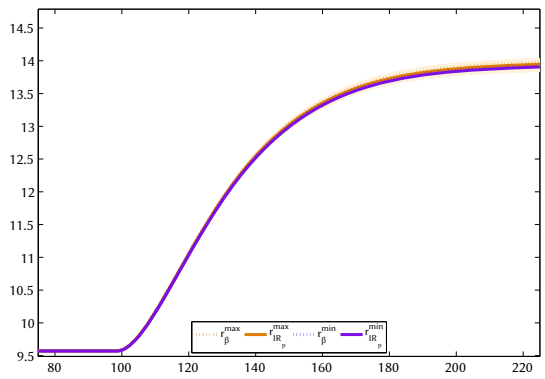

Length $=40,000$

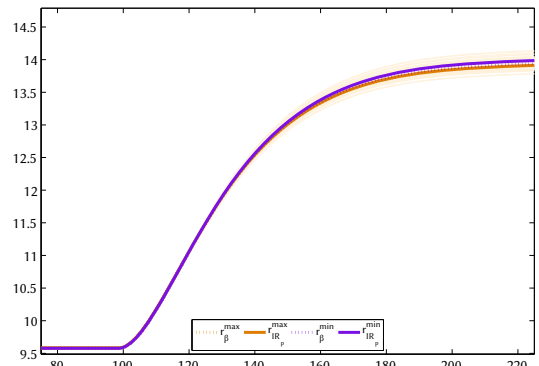

Length $=20,000$

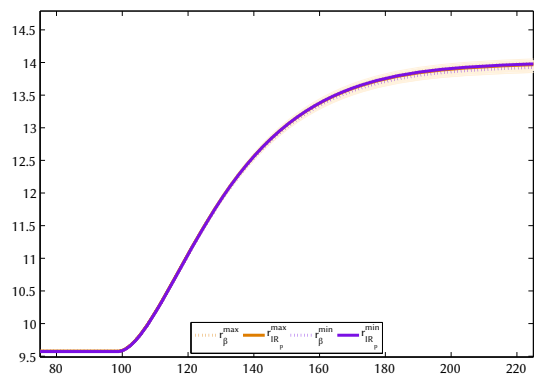

Length $=50,000$

Fig. 5. Responses of $\mathrm{k}$ to a permanent technology shock. SN model. $r_{C}^{\min } / r_{C}^{\max }$ refer to replications minimizing/maximizing $\mathrm{L}^{2}$ distance to reference values ( $C=\tilde{\beta}, \widetilde{R}_{t}$ and $\left.\widetilde{I}_{p}\right)$. (1) Fig. 1. notes applies here.

Firstly, we observe how all responses are qualitatively equal but we have tested that they are statistically different. ${ }^{5}$ The most interesting result, robust across models and types of shock, is the coincidence (tested statistically) of median responses for different lengths. Again, in line with the asymptotic properties of the algorithm, the level of uncertainty is reduced if longer replications are considered. Moreover, the criterion based on median coefficients results non-informative

${ }^{5}$ For the sake of brevity, the results are not included here. They are available upon request in terms of their responses. This finding confirms, again, the noncoincident choices we would achieve for criteria based on coefficients and those based on impulse-response functions.

These criteria do not only help to choose between different solutions but also to measure the robustness of the estimated coefficients. Firstly, the boxplot figures provide summary statistics relative to estimated values. Secondly, the impulse-response functions can be used to construct bands of confidence around the median response, which surely might add robustness to one specific analysis. 


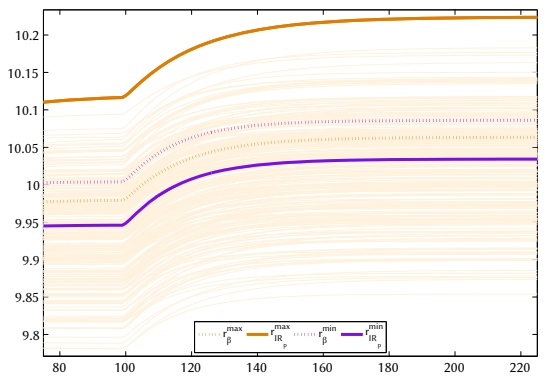

Length $=1,000$

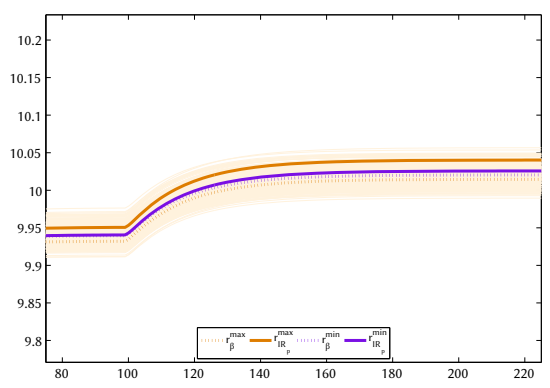

Length $=30,000$

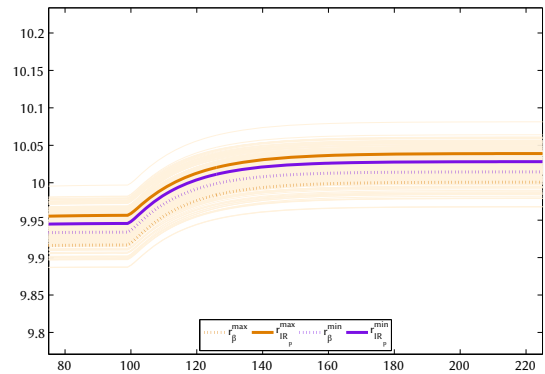

Length $=10,000$

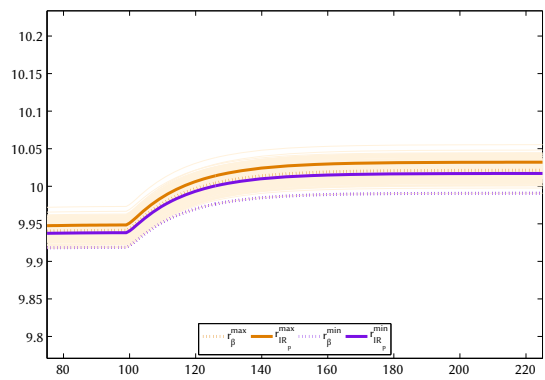

Length $=40,000$

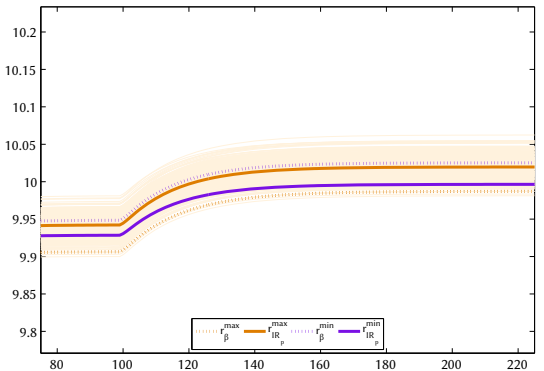

Length $=20,000$

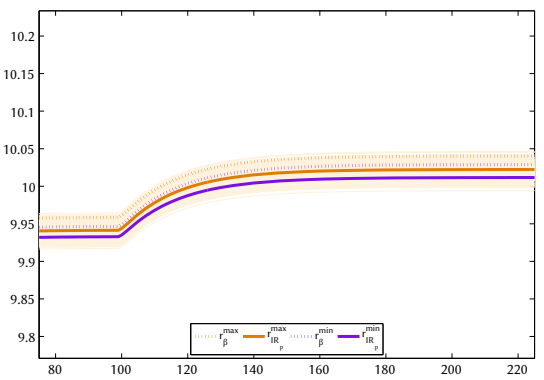

Length $=50,000$

Fig. 6. Responses of $\mathrm{k}$ to a permanent technology shock. $\mathrm{CH}$ model. $r_{C}^{\min } / r_{C}^{\max }$ refer to replications minimizing/maximizing $\mathrm{L}^{2}$ distance to reference values $\left(C=\widetilde{\beta}, \widetilde{R}_{t}\right.$ and $\left.\widetilde{R}_{p}\right) .(1)$ Fig. 1. notes applies here.
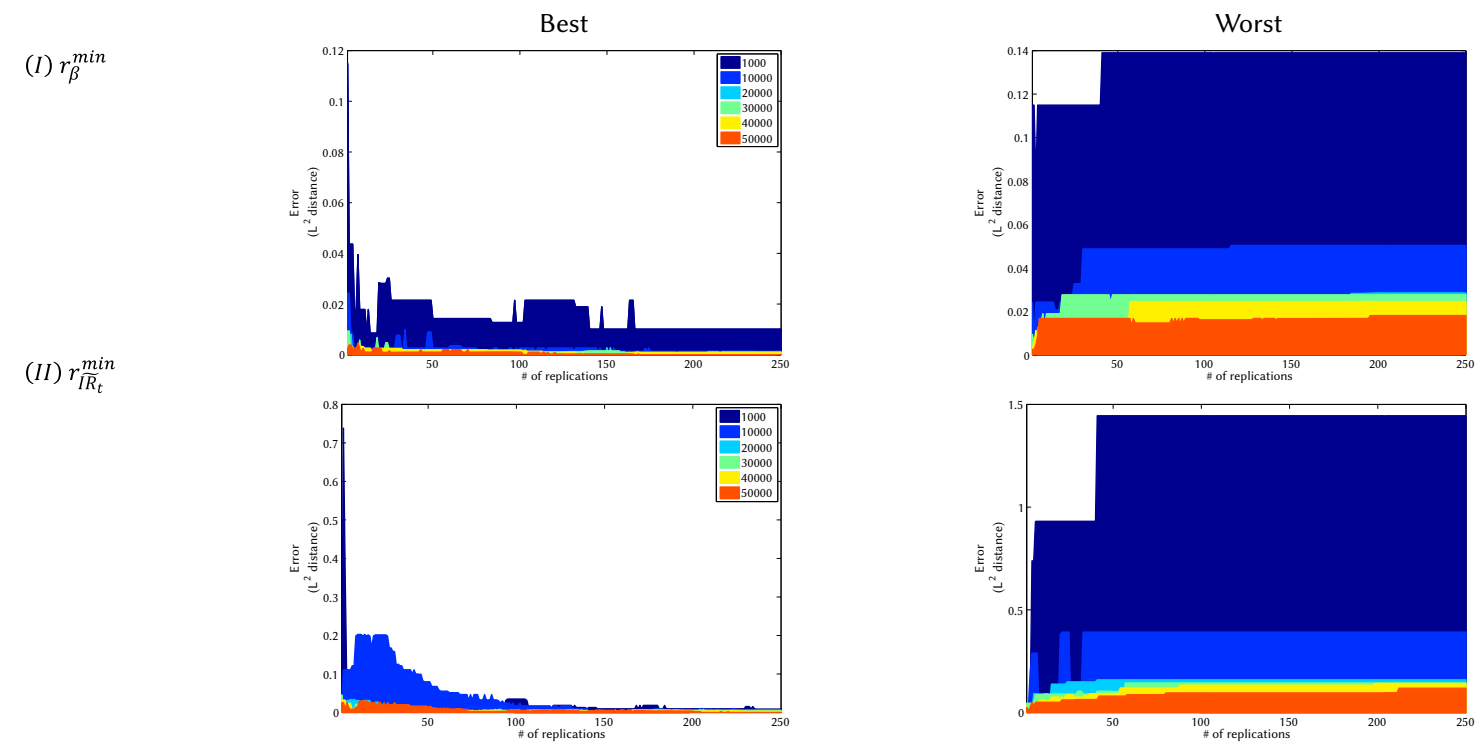

(III) $r_{\overline{I R} p}^{\min }$
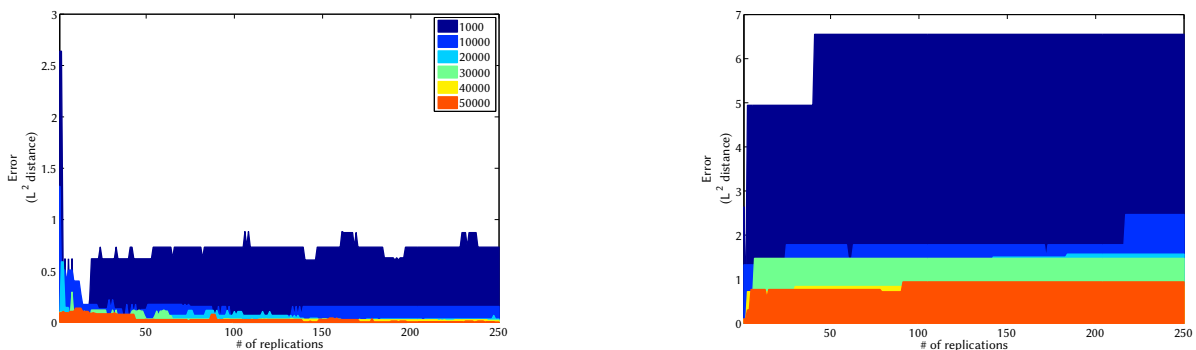

Fig. 7. Relative error with respect to the true solution (Minimal distance to reference values for $\mathrm{T}=50,000$ ). SN model. (II) $r_{\bar{C}}^{\text {min }}$ refers to replications minimizing $\mathrm{L}^{2}$ distance to reference values. (1) Fig. 1. notes applies here.

Another relevant issue to be explored is the sensitivity of these criteria to the number of replications used. In this regard, we compute $\mathrm{L}^{2}$ distance (error) to the replication we would choose according to each criterion but considering a varying number of replications, starting from the assumption that longest length replications are closer to the true solution. The resulting errors are shown in Fig. 7 and Fig. 8. Left (right) panels show how error evolves with the number of replications and the sample size. It can be seen that 75-100 replications 

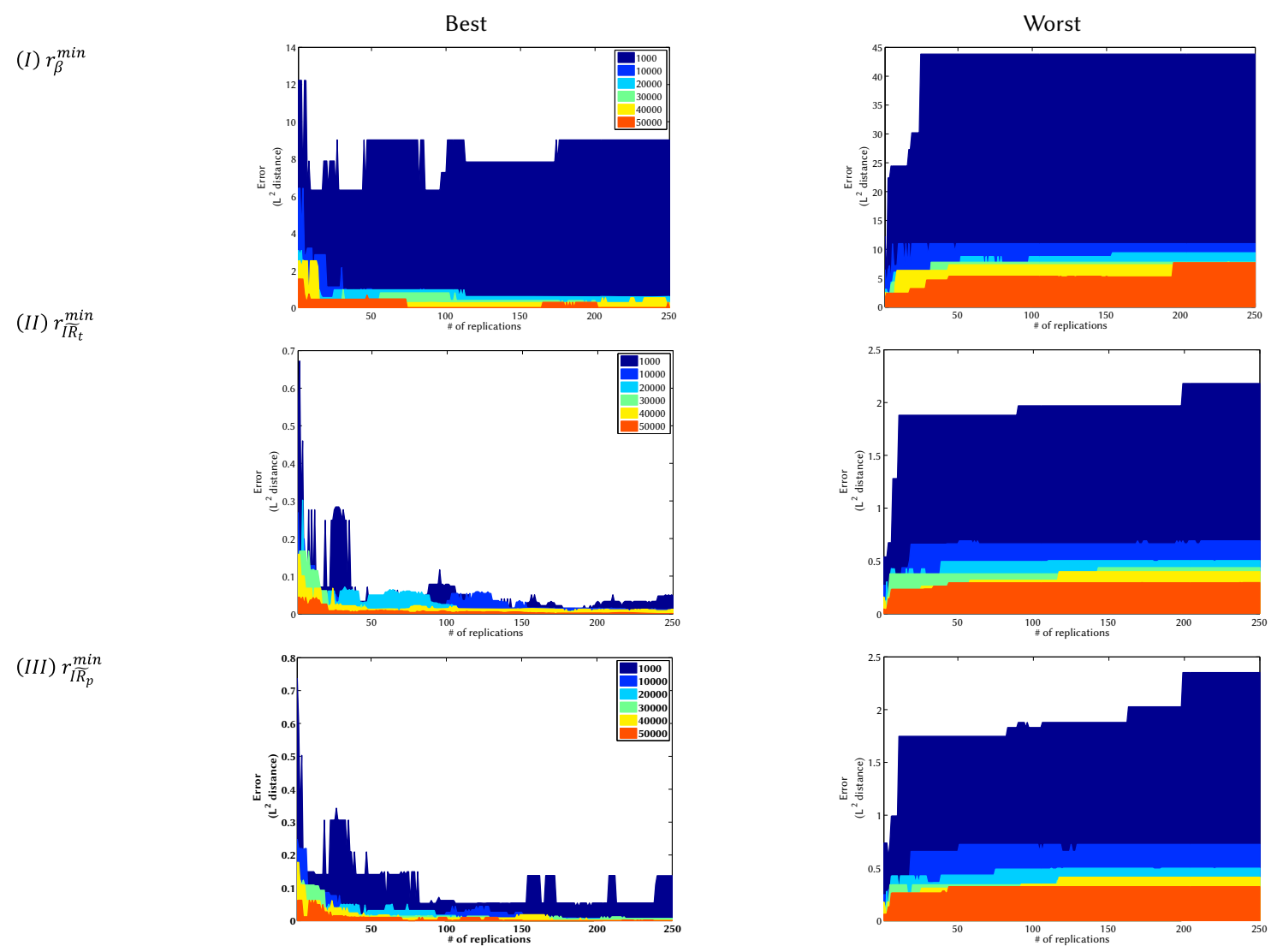

Fig. 8. Relative error with respect to the true solution (Minimal distance to reference values for T=50,000). CH model. (II) $r_{\bar{C}}^{\text {min }}$ refers to replications minimizing $\mathrm{L}^{2}$ distance to reference values. (1) Fig. 1. notes applies here.

are enough to minimize the error and generate the existing variance. With respect to the replication length, no significant improvements in the minimization choice are achieved from considering lengths greater than $10,000 .{ }^{6}$ They also prove that gains in dispersion (a lower distance from the "true" solution to replication maximizing distance to reference values) are obtained mainly from 1,000 to 20,000. After then, error mainly remains at the same values. The latter result mainly confirms our findings regarding the variability of coefficients distributions.

\section{CONCLUSIONS}

We find significant differences among different solutions which clearly establish the relevance of this issue (increasing with the level of complexity of the model). Additionally, robust results are obtained due to the summary statistics we compute from the distributions achieved. By contrast, if only one sufficiently long simulation is considered (what people have commonly done so far), there is no guarantee that a representative solution is obtained (there is heterogeneity among solutions even for $T=50,000$ ).

Moreover, median values are almost equal for different simulation lengths and, therefore, there is no bias in using them. However, the variance among solutions is clearly higher when shorter replications are considered. On the contrary, different criteria rarely accord with their choices. Hence, any decision related to the criterion to be adopted may have consequences on one specific application. However, criteria based exclusively on estimated coefficients ignore the economic performance of these solutions, which represent a significant

${ }^{6}$ We have checked that there are no relevant differences among 5000 and 10000. This information is available upon request. drawback. Note that there is no guarantee of selecting a non-extreme solution if one is finally interested in, for example, observing how will respond our model to a shock (impulse-response functions).

All in all, the median response criterion appears to be the most suitable criterion for several reasons: (i) there is no bias in using shorter simulations (it allows one to face much lower computational costs), (ii) it shows how different solutions behave when a transitory/ permanent shock is applied, and (iii) it provides a band of confidence around the final choice (the level of uncertainty is measured).

Finally, we discuss the number of replications needed to capture the main properties of the distributions under observation. In our exercise, 75-100 replications are enough to minimize the error and generate the existing variance due to the use of shorter replications.

\section{ACKNOWLEDGMENT}

I thank Javier Pérez for his contributions to previous versions of this document and Jesús Rodríguez-López and Alfonso R. Sánchez for helpful comments and discussions. Author acknowledges the financial support of the Spanish Ministry of Science (PID2019-105517RB-I00 and 2006-04803), Ministry of Economy and Competitiveness (ECO201237572) and the Regional Government of Andalusia (SEJ 1512).

\section{REFERENCES}

[1] L. Christiano, and J.M. Fisher, "Algorithms for Solving Dynamic Models with Occasionally Binding Constraints," fournal of Economic Dynamics and Control, vol. 24, pp. 1179-1232, 2000, doi: 10.1016/S01651889(99)00016-0.

[2] W. den Haan and A. Marcet, "Solving the Stochastic Growth Model by Parameterized Expectations," fournal of Business and Economic Statistics, 
vol. 8, pp. 31-34, 1990, doi: 10.1080/07350015.1990.10509770.

[3] W. den Haan and A. Marcet, "Accuracy in Simulations," Review of Economic Studies, vol. 61, pp. 3-17, 1994, doi: 10.2307/2297873.

[4] B.C. Eaves and K. Schmedders, "General equilibrium models and homotopy methods," fournal of Economic Dynamics and Control, vol. 23, pp. 1249-1279, 1999, doi: 10.1016/S0165-1889(98)00073-6.

[5] P. Fackler, "A Matlab solver for Nonlinear Rational Expectations Models," Computational Economics, vol. 26, pp. 173-181, 2005, doi: 10.1007/s10614005-1784-z.

[6] C.B. Garcia and W.I. Zangwill, "Pathways to solutions, fixed points, and equilibria," Prentice- Hall, 1981.

[7] S. Maliar and L. Maliar, "Parameterized expectations algorithm and the moving bounds," Journal of Business and Economic Statistics, vol. 1, pp. 88-92, 2003, doi: 10.1198/073500102288618793.

[8] S. Maliar and L. Maliar, "Parameterized Expectations Algorithm: How to Solve for Labor Easily," Computational Economics, vol. 25, no. 3, pp. 269274, 2005, doi: 10.1007/s10614-005-2224-9.

[9] M. Creel, "Using Parallelization to Solve a Macroeconomic Model: A Parallel Parameterized Expectations Algorithm," Computational Economics, vol. 32, no. 4, 2008, doi: 10.1007/s10614-008-9142-6.

[10] J.B. Taylor and H. Uhlig, "Solving Nonlinear Stochastic Growth Models: A Comparison of Alternative Solution Methods," fournal of Business and Economic Statistics, vol. 8, pp. 1-17, 1990, doi: 10.1080/07350015.1990.10509766.

[11] A. Marcet and D.A. Marshall, "Solving Nonlinear Rational Expectations Models by Parameterized Expectations: Convergence to Stationary Solutions," Institute for Empirical Macroeconomics Discussion Paper 91, 1994, May.

[12] T.F. Cooley, and G. Hansen, "The Inflation Tax in a Real Business Cycle Model," American Economic Review, vol. 79, pp. 733-748, 1989, doi: 10.4324/9780203070710.ch11.

[13] R.Y. Liu, "On a Notion of Data Depth Based on Random Simplices," The Annals of Statistics, vol. 18, pp. 405-414, 1990, doi: 10.1214/aos/1176347507.

[14] J.J. Pérez and A.J. Sánchez-Fuentes, "Alternatives to initialize the Parameterized Expectations Algorithm," Economics Letters, vol. 102, no. 2, pp. 116-118, 2009, doi: 10.1016/j.econlet.2008.11.017.

[15] J.J. Pérez, "A Log-linear Homotopy Approach to Initialize the Parameterized Expectations Algorithm," Computational Economics, vol. 24, pp. 59-75, 2004, doi: 10.1023/B:CSEM.0000038893.70411.f5.

[16] Y. Zuo and R. Serfling, "General Notions of Statistical Depth Function," The Annals of Statistics, vol. 28, pp. 461-482, 2000, doi: 10.1214/ aos/1016218226.

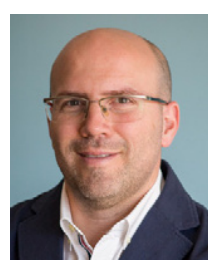

A. Jesus Sanchez-Fuentes

He holds a Bachelor in Mathematics from University of Seville and a Ph.D in Economics from Pablo de Olavide University (with distinctions). He currently works as Associate professor at Complutense University of Madrid where has been appointed as Academic secretary of the Complutense Institute of International Studies (ICEIUCM). He also heads the UCM research group "Family policies", edits the electronic journal on educative innovation "e-pública" and collaborates with the research group Governance and Economics research Network as Research Affiliate. His research areas are mainly public economics and computational economics. 